\title{
Carotenoid-to-bacteriochlorophyll energy transfer through vibronic coupling in LH2 from Phaeosprillum molischianum
}

\author{
Erling Thyrhaug ${ }^{1} \cdot$ Craig N. Lincoln $^{1} \cdot$ Federico Branchi $^{2} \cdot$ Giulio Cerullo $^{2}$ • \\ Václav Perlík $^{3} \cdot$ František Šanda $^{3} \cdot$ Heiko Lokstein ${ }^{4} \cdot$ Jürgen Hauer $^{1}$
}

Received: 31 January 2017 / Accepted: 9 May 2017 / Published online: 18 May 2017

(c) The Author(s) 2017. This article is an open access publication

\begin{abstract}
The peripheral light-harvesting antenna complex (LH2) of purple photosynthetic bacteria is an ideal testing ground for models of structure-function relationships due to its well-determined molecular structure and ultrafast energy deactivation. It has been the target for numerous studies in both theory and ultrafast spectroscopy; nevertheless, certain aspects of the convoluted relaxation network of LH2 lack a satisfactory explanation by conventional theories. For example, the initial carotenoid-to-bacteriochlorophyll energy transfer step necessary on visible light excitation was long considered to follow the Förster mechanism, even though transfer times as short as 40 femtoseconds (fs) have been observed. Such transfer times are hard to accommodate by Förster theory, as the moderate coupling strengths found in LH2 suggest much slower transfer within this framework. In this study, we investigate LH2 from Phaeospirillum (Ph.) molischianum in two types of transient absorption experiments-with narrowband pump and white-light probe resulting in $100 \mathrm{fs}$ time
\end{abstract}

Electronic supplementary material The online version of this article (doi:10.1007/s11120-017-0398-3) contains supplementary material, which is available to authorized users.

Jürgen Hauer

juergen.hauer@tuwien.ac.at

1 Photonics Institute, TU Wien, Gußhausstraße 27, 1040 Vienna, Austria

2 Dipartimento di Fisica, IFN-CNR, Politecnico di Milano, Piazza L. da Vinci, 32, 20133 Milan, Italy

3 Faculty of Mathematics and Physics, Institute of Physics, Charles University, Ke Karlovu 5, 12116 Prague,

Czech Republic

4 Department of Chemical Physics and Optics, Charles University, Ke Karlovu 3, 12116 Praha 2, Czech Republic resolution, and with degenerate broadband 10 fs pump and probe pulses. With regard to the split $\mathrm{Q}_{\mathrm{x}}$ band in this system, we show that vibronically mediated transfer explains both the ultrafast carotenoid-to-B850 transfer, and the almost complete lack of transfer to B800. These results are beyond Förster theory, which predicts an almost equal partition between the two channels.

Keywords LH2 - Ultrafast spectroscopy $\cdot$ Excitation energy transfer $\cdot$ Excitons $\cdot$ Photosynthesis

\section{Introduction}

In the initial steps of photosynthesis, a pigment is excited by absorption of a solar photon. Through a series of excitation energy transfer (EET) steps, this excitation energy is transferred to a photosynthetic reaction center, where conversion into electrochemical energy takes place (Blankenship 2014). Numerous studies using time-resolved spectroscopy have shown that these initial steps of absorption and EET, occurring in adaptable and versatile pigment-protein complexes, proceed on timescales of tens of femtoseconds to hundreds of picoseconds. These pigment-protein complexes and their interactions are heavily reliant on the interplay of structure and function, and ultimately only through the combination of structural biology and ultrafast spectroscopy one can understand the complex energy transfer network and dynamics in photosynthetic systems. An early example of successfully established structure-function relationship is the peripheral light-harvesting antenna complex LH2 found in purple bacteria. With its crystal structure determined as early as $1995, \mathrm{LH} 2$ from the purple bacterium Rhodopseudomonas (Rps.) acidophila is one of the most intensely studied light-harvesting units (Mcdermott 
et al. 1995). It consists of a symmetric array of nine $\alpha / \beta$ apoprotein heterodimers. Each of these units binds three bacteriochlorophyll $a$ (BChl) molecules and one carotenoid. In the fully assembled oligomer, 18 of the BChls are incorporated into a strongly coupled ring-like structure, the $\mathrm{B} 850$ ring, while the remaining nine BChls form the weakly coupled B800 ring. Even though the overall ringlike structure of LH2 complexes shows a high degree of interspecies similarity, significant deviations are observed upon closer inspection. While in Rps. acidophila and Rhodobacter sphaeroides ninefold symmetry is observed, LH2 from Phaeospirillum (Ph.) molischianum shows eightfold symmetry, with BChl rings of 16 and eight pigments, respectively, for B850 and B800. Nevertheless, the absorption spectra in the NIR region, where these $\mathrm{BChl}$ ring structures show their peak absorption, are almost identical for all these species (Herek et al. 2004). A further distinguishing structural feature between these two complexes, as illustrated in Fig. 1, is the orientation of the B800 ring relative to the $\mathrm{B} 850$ ring. While in Rps. acidophila the $\mathrm{B} 800$ $\mathrm{BChl}$ is nearly orthogonal to the $\mathrm{B} 850 \mathrm{BChls}$, the $\mathrm{B} 800$ BChl in Ph. molischianum is rotated in plane by $90^{\circ}$ and tilted by $38^{\circ}$.

Besides these structural differences, a large variety of carotenoids are bound to LH2 complexes extracted from different organisms (Polivka and Sundstrom 2004). Carotenoids can be described as chain-like polyenes with their photophysical properties to a large extent determined by the number of conjugated double bonds, $N$, ranging from 9 to 13 in naturally occurring bacterial complexes. Most of
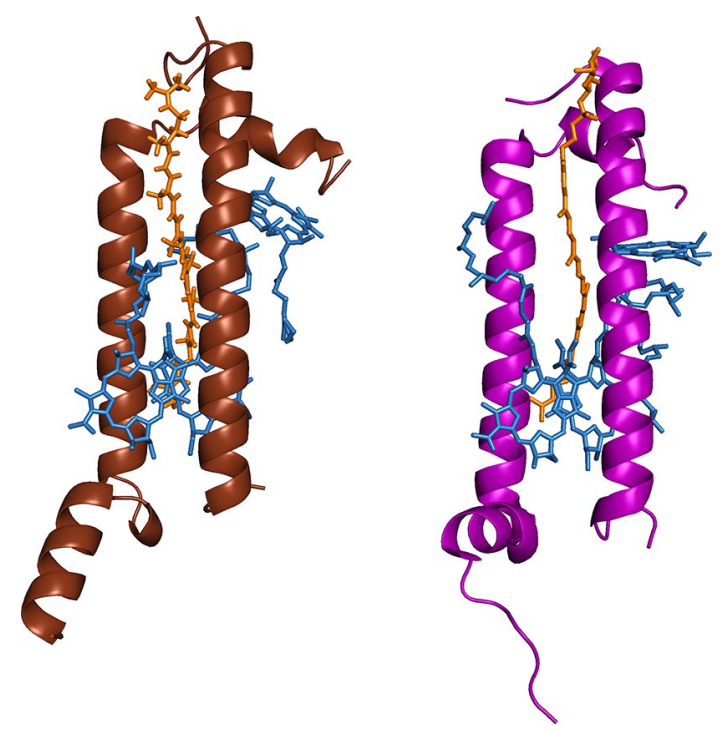

Fig. 1 The LH2 $\alpha / \beta$ heterodimers in the crystal structures of $P h$. molischianum (left) and Rps. acidophila (right). Bacteriochlorophylls are highlighted in blue and carotenoids in orange. Structures from the protein data bank (PDB) the bacterial light-harvesting relies on BChls and their excitonic bands, and while carotenoids can also act as an accessory pigment, their primary task is photoprotection, i.e., the quenching of long-lived and potentially harmful BChl triplet states (Blankenship 2014; Niedzwiedzki et al. 2011). Nonetheless, when acting as light-harvesters in the photosynthetic apparatus of purple bacteria, carotenoids have appreciable absorption in the blue-green spectral region of the solar spectrum. Given the steep distance dependence of known EET mechanisms (Valkunas et al. 2013), it is clear that carotenoids need to be in close proximity of BChls for both EET and photoprotection. The structures compared in Fig. 1 illustrate that both rhodopin glucoside (the carotenoid found in Rps. acidophila) and lycopene (Ph. molischianum) are in van der Waals contact $(<5 \AA)$ with the respective B850 and B800 BChls.

EET from carotenoids to BChls may occur from at least two excited states: the lowest lying singlet state $S_{1}$, which is optically dark for transitions from the ground state $\left(S_{0}\right)$ and the first optically allowed singlet excited state $\left(S_{2}\right)$. These two states differ not only in the character of their optical transitions from the ground state, but also in their lifetimes of tens to hundreds of femtoseconds for $S_{2}$ and a few to tens of picoseconds for $S_{1}$ (Polivka and Sundstrom 2004). The ultrashort lifetime of $S_{2}$ is explained by relaxation to $S_{1}$, with possible involvement of other intermediate states (Polivka and Sundstrom 2009). Upon excitation of the $\mathrm{S}_{2}$ state of a LH2-bound carotenoid, the lowest-energy band formed from $\mathrm{BChl}_{\mathrm{y}}$ transitions in the $\mathrm{B} 850$ ring $\left(Q_{y} B 850\right)$ is populated within several $100 \mathrm{fs}$, which makes $\mathrm{S}_{2}$ a likely donor state despite its ultrashort lifetime (Shreve et al. 1991; Macpherson et al. 2001). For Ph. molischianum, Rondonuwu et al. (2004) found a 48\% EET efficiency from $\mathrm{S}_{2}$ to BChl, while the transfer from $\mathrm{S}_{1}$ appeared to be negligible. The latter is explained by the $S_{1}$-energy of lycopene $(N=11)$ being $12.500 \pm 150 \mathrm{~cm}^{-1}$, which hinders transfer to the almost isoenergetic $\mathrm{Q}_{\mathrm{y}} \mathrm{B} 800$ band, while direct transfer to $\mathrm{Q}_{\mathrm{y}} \mathrm{B} 850$ is unfavorable due to a mismatch of absorption and fluorescence spectra (Billsten et al. 2002a). The same reasoning applies to rhodopin glucoside, which also has 11 double bonds (Polivka et al. 2002).

To describe the EET process from $\mathrm{S}_{2}$ to the bands formed by $\mathrm{BChl} \mathrm{Q}_{\mathrm{x}}$ transitions, given the moderate coupling of approximately $110 \mathrm{~cm}^{-1}$ (Tretiak et al. 2000; Damjanovic et al. 1999), theoretical approaches grounded in perturbative treatment of coupling (Förster theory) have been employed. However, even using sophisticated ab initio estimates of couplings (e.g., the transition density cube method), Förster theory tends to overestimate $\mathrm{S}_{2}$-to-Qx transfer times (Tretiak et al. 2000), which can be as fast as 40 fs for LH2 from Marichromatium purpuratum (Polli et al. 2006) or even 26 fs in the main light-harvesting pigment-protein complex of green plants (LHCII) (Knox 
1999). As such, these extremely fast transfer times seem incompatible with the traditional framework of Förster theory.

Recently, Perlik et al. (2015) described how vibronic coupling successfully explains both dynamical and static spectral properties of a donor-acceptor system closely mimicking the properties of $S_{2}$ (carotenoid) and $Q_{x}(B C h l)$. In this vibronic model, an underdamped vibration located at the carotenoid (donor) was treated on the same footing as electronic degrees of freedom in the system Hamiltonian. This allowed for a discrete vibrational structure of the electronic ground and excited state manifold. Resonance between the donor-acceptor energy gap and transitions to vibronically shifted levels on electronic ground and excited states allowed for dissipation of excess energy during carotenoid-to-BChl transport to the ground-state vibration, which explained the observed ultrafast transfer rates.

The "split" $\mathrm{Q}_{\mathrm{x}}$ band of Ph. molischianum represents an ideal test case for the vibronic coupling model: as the coupling constants between $S_{2}$ and both $Q_{x}$ bands $\left(Q_{x} B 800\right.$ and $Q_{x} B 850$ in Fig. 2) are known (Tretiak et al. 2000), the predictions of this model can be tested for two independent EET channels. To this end, we perform two transient absorption experiments: in the first set of experiments, we excite $\mathrm{S}_{2}$ and use visible- and NIR-supercontinuum probe to study the entire EET network with sub-100 fs time resolution. To accurately characterize the $S_{2}$-to-Qx EET dynamics, which have been observed to proceed on timescales

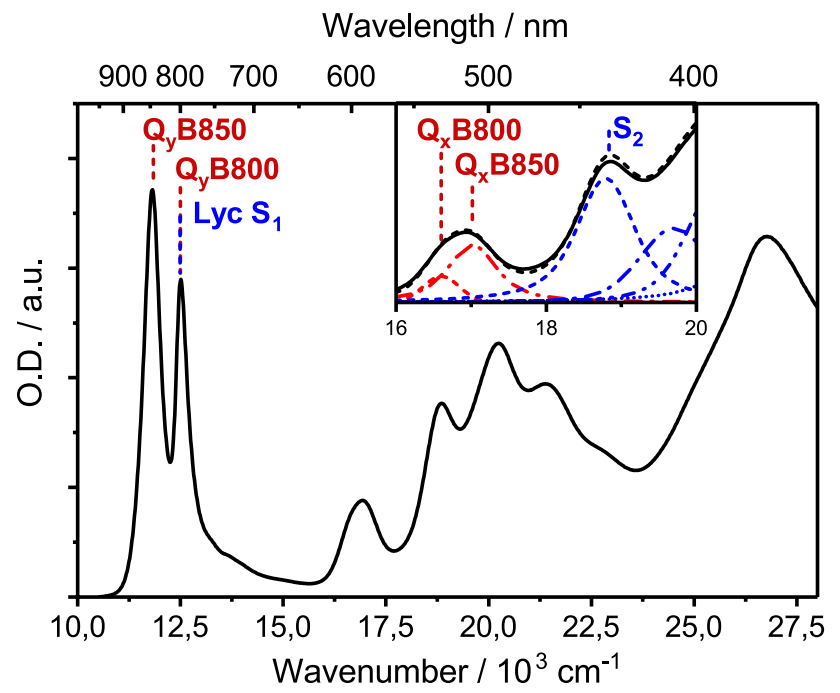

Fig. 2 Normalized absorption spectra of LH2 from Ph. molischianum in buffer solution at room temperature. The $\mathrm{Q}_{\mathrm{y}}$ bands associated with the B800 and B850 rings are labeled in red, while the approximate position of the lycopene $S_{1}$ transition in solution is indicated in blue. Inset: Magnified view of the $\mathrm{BChlQ}_{\mathrm{x}}$ transition region from 16.000 to $20.000 \mathrm{~cm}^{-1}$. The absorption profile can be fit to a "split" $\mathrm{Q}_{\mathrm{x}}$ band (red) and a series of vibronic bands related to the lycopene $\mathrm{S}_{2}$ absorption (blue) well below 100 fs, we additionally perform sub-10 fs transient absorption experiments with visible pulses. By combination of both experiments, along with global (target) analysis, we arrive at a comprehensive picture of energy flow dynamics in Ph. molischianum. We further show that the vibronic coupling model, applied to the LH2 complex, is able to explain both the rate and the directionality of the EET process.

\section{Results and discussion}

The absorption spectrum of LH2 from Ph. molischianum, shown in Fig. 2, is typical for purple bacterial LH2 complexes. Two sharp excitonic transitions, $\mathrm{Q}_{\mathrm{y}} \mathrm{B} 850$ and $\mathrm{Q}_{\mathrm{y}} \mathrm{B} 800$, formed from $\mathrm{Q}_{\mathrm{y}}$ transitions of the two $\mathrm{BChl}$ rings are observed in the near-infrared. The associated out-ofplane $\mathrm{BChlQ}_{\mathrm{x}}$ transitions appear as broad and relatively weak features around $16.500 \mathrm{~cm}^{-1}(\sim 600 \mathrm{~nm})$, and show properties similar to monomeric BChl (Blankenship 2014). In most LH2 complexes, e.g., from Rps. acidophila or $R b$. sphaeroides, the $\mathrm{Q}_{\mathrm{x}}$ transitions are essentially degenerate, resulting in a close-to-symmetric absorption band (Rondonuwu et al. 2004; Billsten et al. 2002a; Cong et al. 2008; Christiana et al. 2009). In contrast, the $\mathrm{Q}_{\mathrm{x}}$ band of $P h$. molischianum is clearly asymmetric, and is best described by two overlapping transitions with slightly different central frequencies $\left(17.030\right.$ and $\left.16.600 \mathrm{~cm}^{-1}\right)$, as demonstrated in the inset of Fig. 2. The higher energy of these transitions contains approximately twice the oscillator strength of the lower transition, suggesting that it is the $Q_{x}$ band associated with the B850 ring. This energetic splitting suggests that the $Q_{x}$ transitions of B800 and B850 BChls experience different coupling to the surrounding protein in LH2 of $P h$. molischianum, while the two environments appear to be equivalently polar for e.g., Rps. acidophila. Indeed, one finds that a more polar aspartate residue is the $\mathrm{Mg}$ ligand in Ph. molischianum but a formyl-methionine in Rps. acidophila (Koepke et al. 1996).

At excitation energies higher than approximately $18.000 \mathrm{~cm}^{-1}$ in the visible range, optical transitions are assigned predominantly to the progression of carotenoid $\mathrm{S}_{0}-\mathrm{S}_{2}$ vibronic states. As coupling between carotenoids and BChls in LH complexes is relatively weak, excited states are largely localized on one type of pigment. The $\sim 1000 \mathrm{~cm}^{-1}$ red-shift of the carotenoid absorption observed upon incorporation into LH2 is thus not due to electronic coupling, but rather interaction with the protein pocket (Herek et al. 2004). As such, excitation below approximately $18.000 \mathrm{~cm}^{-1}(555 \mathrm{~nm})$ will result in direct excitation of BChl states, while in the $18-22.000 \mathrm{~cm}^{-1}$ range excitation will predominantly result in population of excited states of lycopene. 


\section{Excitation energy transfer dynamics}

In order to investigate the relaxation processes in LH2 from $P h$. molischianum after carotenoid excitation, we perform a series of transient absorption (TA) experiments. In Fig. 3a the TA spectra using narrowband excitation and supercontinuum probe (see "Experimental" section for details) are presented as a two-dimensional probe frequency versus time map. The overall relaxation dynamics are multi-exponential and involve decay times spanning from tens of femtoseconds to hundreds of picoseconds. The relatively weak BChl-lycopene coupling, however, enables us, to a good approximation, to analyze the complex data in terms of transitions associated with lycopene- and BChl-related states. In particular, it allows us to use the response of isolated lycopene as a reference in the interpretation of relaxation dynamics, greatly facilitating the task of assigning spectral signatures to electronic states.

To aid this assignment, we perform global analysis on the data, using a total of six exponential components to describe the dataset (Fig. 4). The principles of global analysis have been explored in great detail elsewhere (Lincoln et al. 2016; van Stokkum et al. 2004). We show the decay-associated spectra (DAS, parallel decay model) and the evolution-associated differential spectra (EADS, sequential model) in Fig. 4. The quality of the fit can be assessed in Fig. 3c, and the spectra retrieved from the sequential model (evolution-associated difference

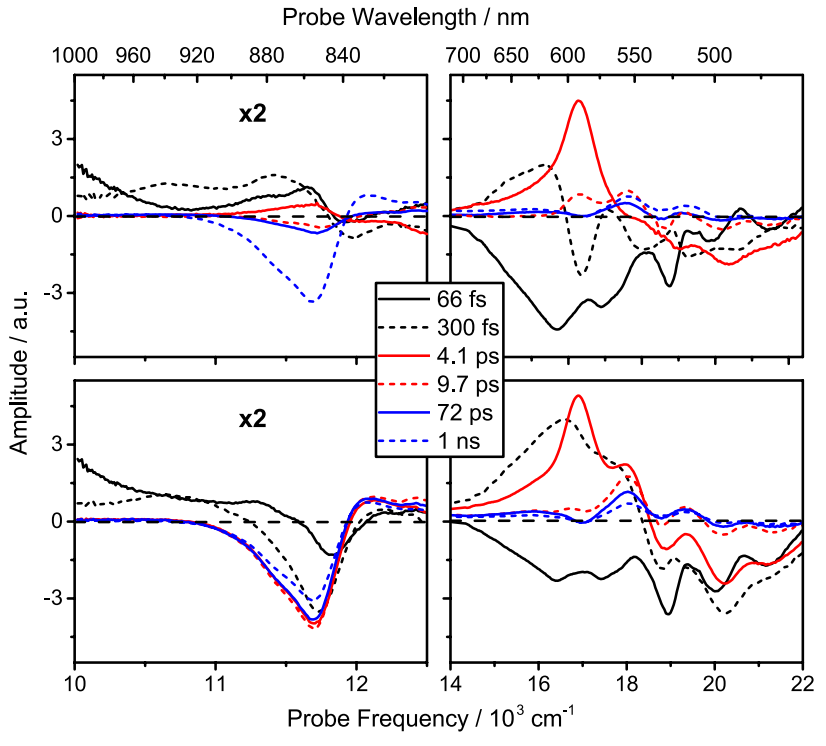

Fig. 4 Decay-associated spectra of LH2 from Ph. molischianum in the near-infrared (left) and visible (right) spectral region. Note the rescaling of the near-infrared amplitudes. Top DAS (parallel decay model), bottom EADS (sequential decay model). The amplitude of the $66 \mathrm{fs}$ component in all panels is divided by 2 in order to facilitate comparison of spectral shapes between components

spectra, EADS) are in good agreement with the previously reported spectra (Rondonuwu et al. 2004; Billsten et al. 2002a, b).

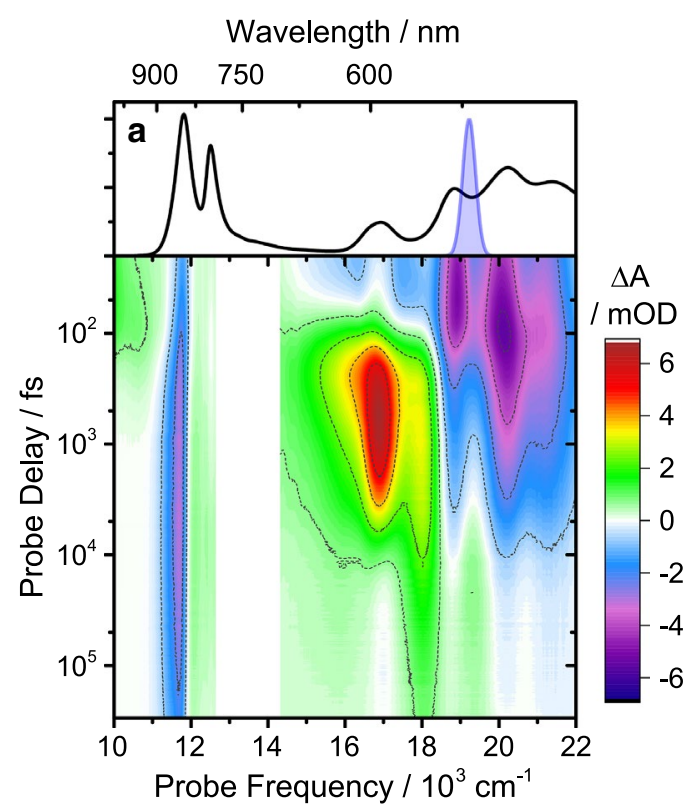

Fig. 3 a Linear absorption spectrum and 2D time/frequency transient absorption map following excitation of the lycopene $\mathrm{S}_{0}-\mathrm{S}_{2}$ transition. The excitation pulse spectrum is indicated by the blue shaded area.

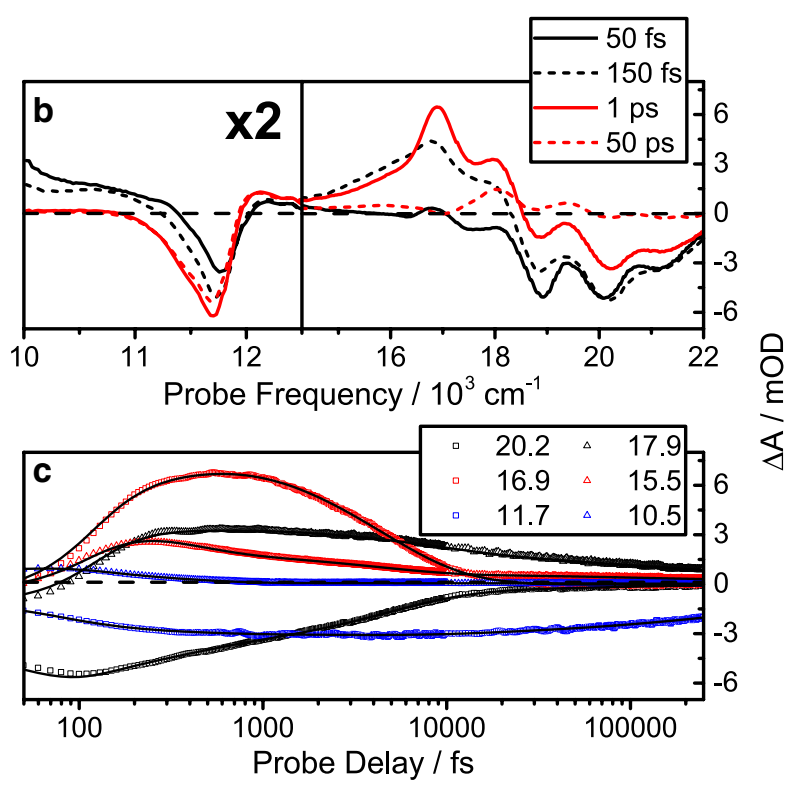

b Transient absorption spectra at selected delay times. c Kinetics at selected probe frequencies (symbols) overlaid with fits from a global exponential decay model (solid lines). Units are in $10^{3} \mathrm{~cm}^{-1}$ 
In short, in the $14-22.000 \mathrm{~cm}^{-1}$ region essentially all spectral features are attributable to lycopene localized states. The first bright state, the $\mathrm{S}_{2}$ state of lycopene, has a lifetime of $66 \mathrm{fs}$ and exhibits strong ground-state bleach (GSB) and stimulated emission (SE) features. This is followed by the vibrationally hot $\mathrm{S}_{1}$ state (hot- $\mathrm{S}_{1}$ ), which cools on a 300 fs timescale to the equilibrated $S_{1}$ state. The lifetime of this state, $4.1 \mathrm{ps}$, is in good agreement with the literature values for lycopene in solution (Kosumi et al. 2009; Billsten et al. 2002b). Both of these states show strong excited state absorption (ESA) contributions between 14 and $19.000 \mathrm{~cm}^{-1}$ in addition to the $S_{2}$ GSB. The following three retrieved spectra, with time constants 9.7, 72 ps and $1 \mathrm{~ns}$, all exhibit an ESA feature at $18.000 \mathrm{~cm}^{-1}$ and only a weak $\mathrm{S}_{2}$ GSB contribution. This feature, which can also be seen in the hot- $S_{1}$ and $S_{1}$ spectra, has been assigned to a carotenoid triplet, a triplet precursor, the so-called $\mathrm{S}^{*}$ state, recently to a field induced shift of the $S_{2}$ GSB (Herek et al. 2004; Niedzwiedzki et al. 2016) and combinations thereof. A full analysis of the feature is beyond the scope of this paper, and we will from here on refer to it as $\mathrm{S}^{*}$ (Gradinaru et al. 2001; Christensson et al. 2009; Balevičius et al. 2015, 2016). Additional features attributed to the carotenoid are two ESA bands in the $10-12.000 \mathrm{~cm}^{-1}$ region. One of these, having a $66 \mathrm{fs}$ lifetime, is assigned to $\mathrm{S}_{2}$-to- $\mathrm{S}_{\mathrm{m}}$ transitions (Christensson et al. 2010), while the second with lifetime of $300 \mathrm{fs}$ is tentatively assigned to hot $\mathrm{S}_{1}$-to- $\mathrm{S}_{\mathrm{n}}$ transitions (Andersson and Gillbro 1995).

Beyond these minor ESA features, the $11-13.000 \mathrm{~cm}^{-1}$ NIR region of the spectrum is dominated by BChl features, in good agreement with earlier reports (Ihalainen et al. 2001). In both the early-time raw data (Fig. 3c) and in the first 66 fs components in the global decay models (Fig. 4), there is a pronounced GSB corresponding to the $\mathrm{Q}_{\mathrm{y}} \mathrm{B} 850$ absorption. We attribute this to excitation of highlying states associated with the $\mathrm{B} 850$ ring $\left(\mathrm{Q}_{\mathrm{x}}\right.$ or $\left.\mathrm{B}\right)$, and their strong coupling to $\mathrm{Q}_{\mathrm{y}} \mathrm{B} 850$. The following time component (300 fs) spectra are significantly red-shifted, with a weak ESA feature towards the blue edge. This is due to a population appearing in the $\mathrm{Q}_{\mathrm{y}} \mathrm{B} 850$ band (Rondonuwu et al. 2004), resulting in SE and ESA features related to the band in addition to the already present GSB. Hence the rapid buildup of the excited state population in the $Q_{y} B 850$ band proceeds on a timescale of 100-300 fs. On longer timescales, illustrated by the spectra at 1 ps (Fig. 3c) and the 4.1 ps global decay component (Fig. 4), we can observe only a minor increase in this feature. This implies that $\mathrm{Q}_{\mathrm{y}} \mathrm{B} 850$ excited state population is predominantly formed by transfer from $\mathrm{S}_{2}$, rather than the slower channels involving transfer via $S_{1}$ states. The concomitant $Q_{x} B 850$ GSB signal is almost impossible to see without de-convolving the spectra into their constituent components due to the much stronger opposing ESA from the carotenoid. One can see it beginning to appear for delays longer than $50 \mathrm{ps}$ though it is still not fully resolved.

Importantly, we find no indication of significant $\mathrm{Q}_{\mathrm{y}} \mathrm{B} 800$ population during the relaxation process. This could partly be due to its transient nature, as it is known to relax to $\mathrm{Q}_{\mathrm{y}} \mathrm{B} 850$ on $a \sim 1$ ps timescale (Billsten et al. 2002a; Theiss et al. 2008), and to the spectral overlap with $Q_{y} B 850$ ESA. Notwithstanding, it remains less than expected from other LH2, e.g., Allochromatium vinosum (Niedzwiedzki et al. 2012) and $R b$. sphaeroides (Cong et al. 2008).

The $\mathrm{Q}_{\mathrm{y}} \mathrm{B} 850$ formation kinetics substantiates this observation. In LH2 from Allochromatium vinosum and $R b$. sphaeroides, the formation dynamics of the $\mathrm{Q}_{\mathrm{y}} \mathrm{B} 850$ band has multiple components, corresponding to internal $\mathrm{Q}_{\mathrm{x}} \mathrm{B} 850$-to- $\mathrm{Q}_{\mathrm{y}} \mathrm{B} 850$ relaxation, inter-ring $\mathrm{Q}_{\mathrm{y}} \mathrm{B} 800-\mathrm{Q}_{\mathrm{y}} \mathrm{B} 850$ $\mathrm{EET}$, and transfer from the carotenoid $\mathrm{S}_{1}$ state (likely via the $\mathrm{Q}_{\mathrm{y}} \mathrm{B} 800$ band) (Cong et al. 2008). Although the latter two processes are significant in the dynamics of these LH2 complexes, they are relatively slow, with time constants in the range of approximately $800-1000$ fs and 4-5 ps, respectively (Macpherson et al. 2001; Billsten et al. 2002a; Polivka et al. 2007) depending on the organism. In stark contrast, the $\mathrm{Q}_{\mathrm{y}} \mathrm{B} 850$ kinetics displayed in Fig. 3 are simple and strongly dominated by the 66 fs buildup and the slow mono-exponential ground-state recovery on a timescale long compared to the time window probed here. The implication of this observation is that neither the $S_{2}$-to-B800 nor the $S_{1}$-to-B800 relaxation channels contribute significantly to light-harvesting of the complex upon green-light excitation. Overall, these observations strongly suggest that the initial $S_{2}$ population transfers, with essentially equal probability, either to states related to the B850 ring or to the $S_{1}$ state. As $\mathrm{S}_{1}$-to-BChl transfer is inefficient, it appears that on "green-light" excitation, neither lower-energy carotenoid states nor the B800 ring contributes to functional light-harvesting (Damjanovic et al. 1999).

\section{Ultrafast dynamics in the carotenoid/ $Q_{x}$ spectral region}

The narrowband pump-supercontinuum probe studies described above demonstrate that, within the limited time resolution provided by the experiment, the effective carotenoid $S_{2}$-to- $Q_{X} B 850$ transfer proceeds on a timescale of $100 \mathrm{fs}$ or faster. Moreover, the $\mathrm{Q}_{\mathrm{y}} \mathrm{B} 800-\mathrm{Q}_{\mathrm{y}} \mathrm{B} 850$ and $\mathrm{S}_{1}-\mathrm{Q}_{\mathrm{y}} \mathrm{B} 850$ transfer channels present in LH2 from a number of other organisms (Mirkovic et al. 2017) are also clearly shown to be largely insignificant in visible range light-harvesting in Ph. molischianum. However, since the timescale of the rapid rise of $\mathrm{Q}_{\mathrm{y}} \mathrm{B} 850$ is considerably shorter than the instrument response function, it was 
not possible to reliably assess the underlying mechanism behind the suppression of the $\mathrm{S}_{2}-\mathrm{Q}_{\mathrm{y}} \mathrm{B} 800$ channel.

In order to characterize the initial carotenoid relaxation, we perform broadband femtosecond TA using $\sim 10$ fs pulses centered around $17.500 \mathrm{~cm}^{-1}$. Figure 5 shows the employed laser spectrum along with the corresponding LH2 absorption spectrum. The population relaxation dynamics are in good qualitative agreement with the results from the narrowband experiment, with preserved spectral lineshapes for $\mathrm{S}_{2} \mathrm{GSB}$ and SE, hot- $\mathrm{S}_{1}$ ESA, and the cooled $S_{1}$ ESA with a pronounced $S^{*}$ feature (Fig. 5). The retrieved lifetime for $S_{2}$ is considerably shorter than that from narrowband excitation however, and we observe also the GSB and SE features related to the $\mathrm{Q}_{\mathrm{x}} \mathrm{BChl}$ bands at early times due to their direct excitation by the broadband pulse.

The 38 fs time constant for carotenoid $\mathrm{S}_{2}$ decay in LH2, as opposed to $110 \mathrm{fs}$ in lycopene in hexane (Billsten et al. 2002b), implies that the total time constant for $\mathrm{S}_{2}$-to-BChl transfer is approximately $60 \mathrm{fs}$, in agreement with the observation of $\mathrm{Q}_{\mathrm{y}} \mathrm{B} 850$ population within the instrument response in the narrowband experiment. Such fast carotenoid-to-BChl transfer times have been reported also for other light-harvesting antenna systems (Polli et al. 2006; Perlik et al. 2015); however, they are too fast to be reconcilable with dipole-dipole coupling assuming a Förster mechanism (Krueger et al. 1998). It was suggested that the involvement of vibrations and vibronic mixing is of importance for EET processes in LH2 (Perlik et al. 2015). Indeed, our experimental observations suggest that vibrational motion is significantly involved in the energy transfer dynamics, as explained in the following section.

\section{Coherent dynamics in the carotenoid $-Q_{X}$ spectral region}

Clear and persistent oscillations-vibrational quantum beats (QBs) - in the kinetics can be observed at several probe energies after broadband excitation. Analyzing these signals, assigning their physical origin, and determining their (possible) relevance to biological function are some of the major challenges in current research into light-harvesting mechanisms (Fuller et al. 2014). We proceed by isolating the oscillatory components by subtraction of the globally fitted population dynamics from the raw data leaving only purely oscillatory residuals, similar to the work of Lincoln et al. (2016). A Fourier transform of the delay time axis of these residuals yields the 2D map shown in Fig. 6a, where the QB amplitude is plotted as a function of QB frequency and probe frequency. From this map, we can learn not only which QB frequencies are present (and their relative intensities), but also at which probe energies they contribute to the signals. From the integrated amplitude spectrum (IAS) shown in Fig. 6b, generated by integrating the map in Fig. 6a over all probe energies, we identify two particularly prominent modes. These appear with frequencies of $\sim 1100$ and $\sim 1465 \mathrm{~cm}^{-1}$, and can be assigned to wellknown ground-state modes of carotenoids (Merlin 1985) though with an approximately $-50 \mathrm{~cm}^{-1}$ offset. We note

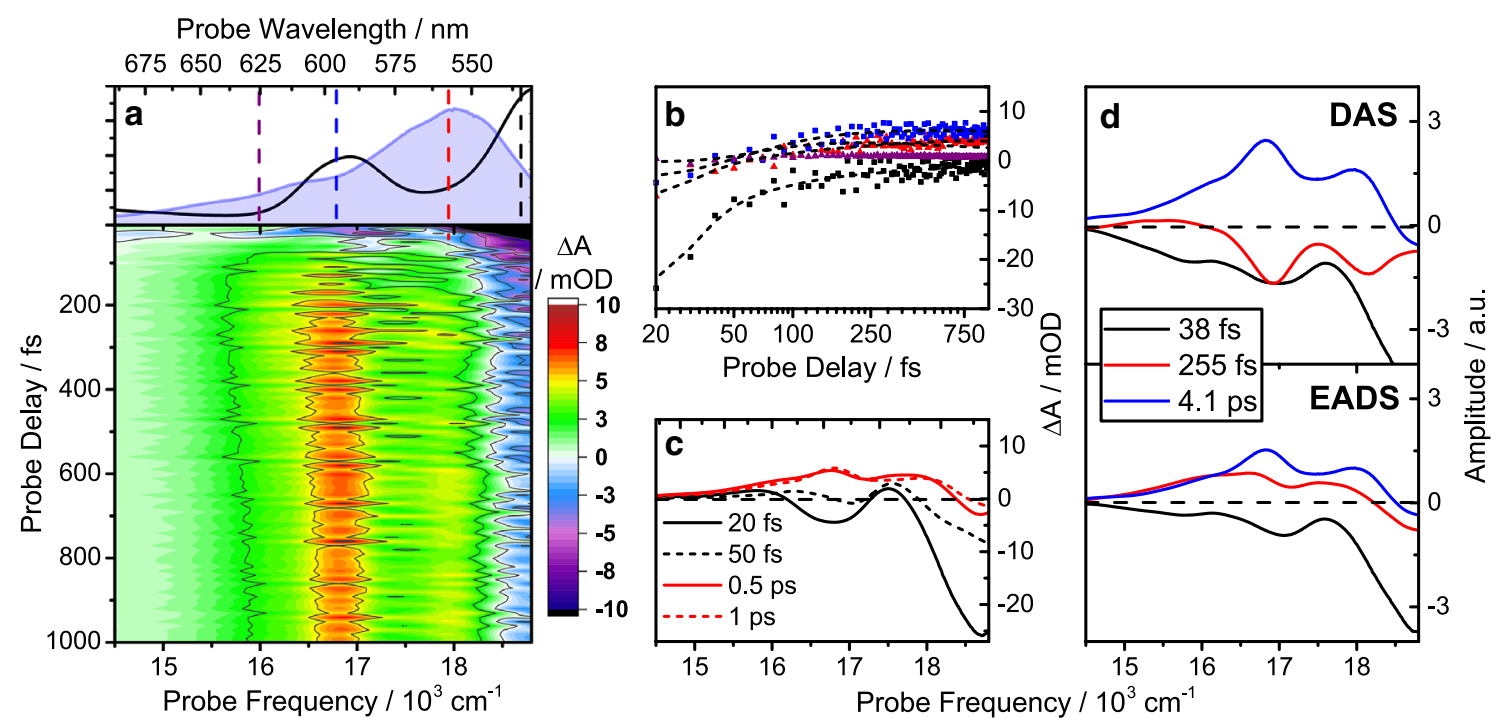

Fig. 5 a Linear absorption spectrum (black line) and laser spectrum (shaded blue area), and 2D time/frequency transient absorption map. b Transient absorption kinetics (symbols) overlaid with the traces from a global fit to the data (dashed lines) at the probe frequencies marked with vertical dashed lines in (a). c Transient absorption spectra at selected probe delays. d Decay-associated spectra (see text for details). The amplitude of the 38 fs component has been halved to facilitate the comparison of spectral shapes 
Fig. 6 a Absorption spectrum (top) and Fourier transform amplitudes of the residuals after subtraction of exponential population dynamics as a function of probe and Fourier transform frequency. b Integration of the data in a over all probe frequencies yields the impulse stimulated Raman spectrum. c Probe frequency dependence of the amplitudes of the two dominating modes (solid lines). The total absorption spectrum and the $\mathrm{Q}_{\mathrm{x}} \mathrm{B} 850$ absorption (black and blue dashed lines) are superimposed for comparison

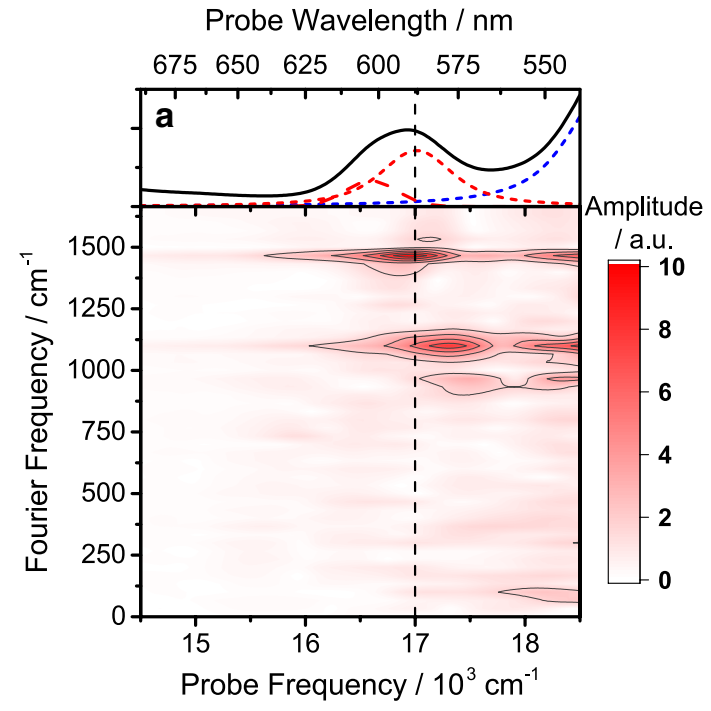

that the IAS is related to the resonance Raman spectrum in the case where QBs originate primarily from groundstate contributions, although the relationship is not entirely trivial (Liebel et al. 2015). Plotting the amplitude of these vibrational modes individually as a function of probe energy yields the spectrum in Fig. 6c. For both modes, we observe two amplitude maxima: one approaching the $\mathrm{S}_{2}$ absorption maximum and one red-shifted by the vibrational frequency relative to a zero-phonon line estimated to be near $18.400 \mathrm{~cm}^{-1}$ (see SI). This is exactly the amplitude pattern expected from consideration of ground-state coherent pathways (i.e., impulsive stimulated Raman scattering) in this system (Lincoln et al. 2016; Jumper et al. 2016). The crucial observation for interpretation of the physical significance of these modes, beyond them being simple ground-state vibrations, is the overlap between the lowerenergy amplitude maximum of the $1465 \mathrm{~cm}^{-1}$ mode and the $\mathrm{Q}_{\mathrm{x}} \mathrm{B} 850$ band. As a result, the strongly allowed carotenoid $S_{2}-S_{0}(0-1)$ transition is in essentially perfect resonance with the $\mathrm{Q}_{\mathrm{X}} \mathrm{B} 850$ transition. This is a requirement for the vibronically mediated transfer mechanism described in detail elsewhere (Perlik et al. 2015), which will be summarized and applied to LH2 from Ph. molischianum in the following section.

\section{The mechanism of excitation energy transfer}

Both low- and high-time resolution TA experiments consistently indicate ultrafast relaxation of the carotenoid $\mathrm{S}_{2}$ state in conjunction with strongly preferential sub-100 fs population of states associated with the $\mathrm{B} 850$ ring. These observations stand in - both quantitative and qualitativecontrast to the calculated relaxation dynamics in this LH2 complex (Tretiak et al. 2000; Damjanovic et al. 1999), where transfer to the $\mathrm{B} 800$ and $\mathrm{B} 850$ rings was suggested to be approximately equal and to proceed on timescales of 200-300 fs. Notably, a common feature of the models used in these earlier theoretical studies is that they rely on essentially Förster-type resonant EET. The TA data, on the other hand, suggest that vibrational states may have nontrivial involvement in the EET process. As vibronic models typically predict significant speed up of EET around excitonic-vibrational resonance as compared to Förster, explicitly including the vibrations in the model thus appears necessary.

In a recent study, Perlik et al. (2015) showed that ground-state vibrations in a synthetic mimic of the LH2 complex heavily influence the observed EET rates under the appropriate resonance conditions. We here apply the vibronic approach (Perlik and Sanda 2016) to LH2 itself by extending the exciton part of the model in Perlik et al. (2015) to contain the two $Q_{x}$ states associated with the $\mathrm{BChl}$ rings along with the $\mathrm{S}_{2}$ state of the carotenoid (see supporting information and Perlik and Sanda 2016 for details). Further, in order to investigate the possible contributions of vibrational states, we explicitly include both of the strongly active ground-state vibrational modes at $\sim 1500$ and $\sim 1100 \mathrm{~cm}^{-1}$ observed in TA experiments. The energy difference between $\mathrm{Q}_{\mathrm{x}} \mathrm{B} 850$ and $\mathrm{Q}_{\mathrm{x}} \mathrm{B} 800$ is fixed to the experimental value, and we calculate the $S_{2}$-to- $Q_{x}$ transfer time (defined as the time needed for the $S_{2}$ population to reach 1/e its initial size) as a function of lycopene-BChl coupling $J$ and the $\mathrm{S}_{2}-\mathrm{Q}_{x} \mathrm{~B} 800$ energy detuning $d E$. The results of the calculation are depicted in Fig. 7a.

The dependence on coupling strength is simple, showing a monotonic decrease of transfer time with increasing $J$ as expected. The energy detuning dependence, on the other hand, shows much more complex behavior, with alternating regions of fast and slow transfer. This complex dependence 


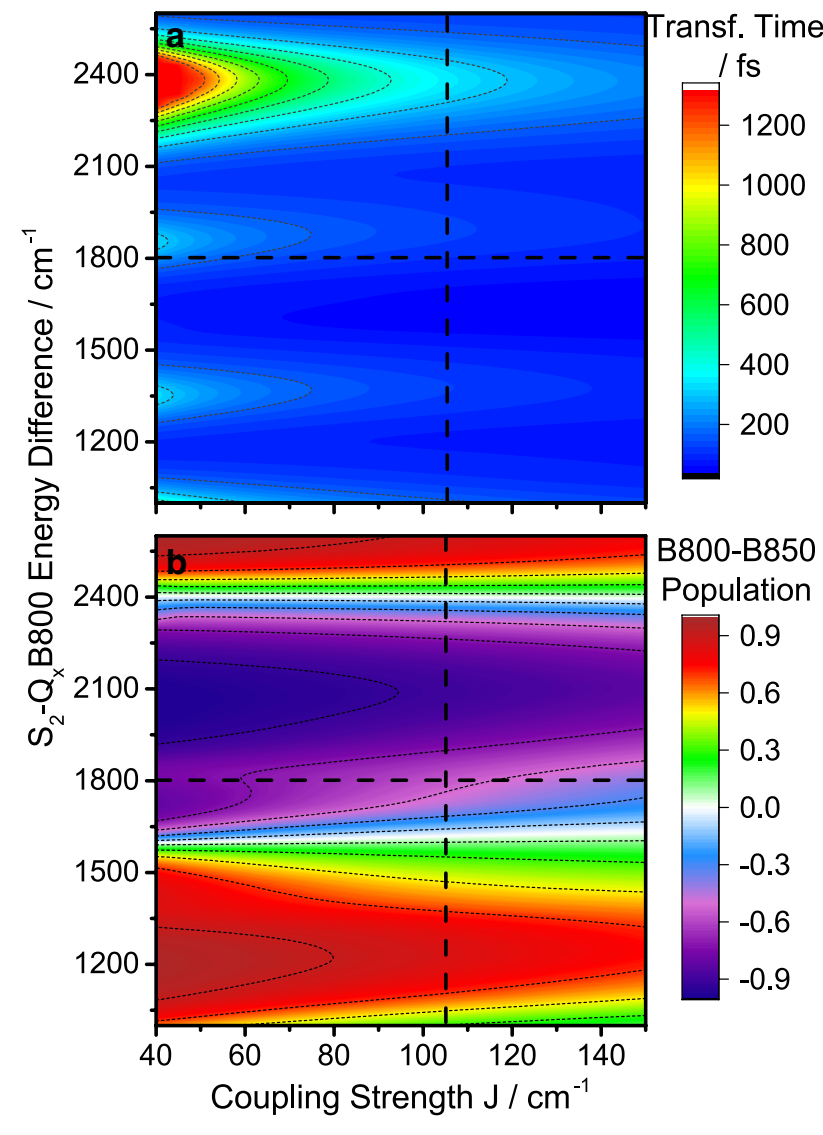

Fig. 7 a $S_{2}$ to $B_{C h l Q_{x}}$ transfer time as a function of level splitting and coupling strength (See text for details) b The normalized population difference between the $\mathrm{B} 800$ and $\mathrm{B} 850$ band at the transfer time $t$ (where the population of $\mathrm{S}_{2}$ is reduced to 1/e of its initial value), as a function of level splitting and coupling strength. Dashed lines correspond to the observed carotenoid $\mathrm{S}_{2}$-B800 level splitting and the coupling strength used in our calculations

on $d E$ can be understood by identifying the most prominent relaxations pathways: In both experiment and model the majority of initial $\mathrm{S}_{2}$ population is generated close to the zero-phonon line, and excitation energy is subsequently transferred from this vibrationally cold $\mathrm{S}_{2}$ state downwards to the energetically closest $\mathrm{BChl}$ eigenstate. Depending on $d E$, the eigenstate nearest to the vibrationally relaxed $\mathrm{S}_{2}$ can be localized on either B800 or B850 (see SI Fig. 1). Minima along the $d E$ axis of Fig. 7a (i.e., regions of fast transfer) appear as the initially excited $\mathrm{S}_{2}$ state of lycopene comes into resonance with a state containing electronically excited $\mathrm{BChl}_{\mathrm{x}}$ and excited carotenoid ground-state vibrations (Perlik et al. 2015).

Applying the experimentally observed energy levels splitting and the lycopene-BChl coupling strengths calculated by Tretiak et al. (2000) as parameters in the model lets us identify the expected transfer times for LH2, denoted by dashed lines in Fig. 7a. With these parameters, the $\mathrm{S}_{2}$ state is close to resonant with $\mathrm{Q}_{\mathrm{x}} \mathrm{B} 850$ and a $1500 \mathrm{~cm}^{-1}$ vibration, while all $\mathrm{Q}_{\mathrm{x}} \mathrm{B} 800$ eigenstates are far out of resonance. As a result, transfer is strongly directional towards the B850 ring, as shown in Fig. 7b. The calculations result in a transfer time of approximately $100 \mathrm{fs}$ and a branching ratio of approximately 3.5 in favor of $\mathrm{Q}_{\mathrm{x}} \mathrm{B} 850$ transfer. This is close to the experimental observations, thus showing that the theoretical approach provides a plausible mechanism to explain both the extremely fast $\mathrm{S}_{2}$-to-BChl transfer and the strong preference for transfer towards the $\mathrm{B} 850$ ring.

\section{Conclusions}

We employed visible pump, visible/NIR white-light probe spectroscopy to monitor the entire carotenoid-to-BChl energy transfer in LH2 from $P h$. molischianum and found that the B800 ring is almost entirely avoided in the energy deactivation network of the complex. To further investigate this intriguing finding, we used sub-10 fs pulses for both pumping and probing and observed extremely fast transfer from $\mathrm{S}_{2}$ to QxB850. The analysis of pronounced vibrational quantum beats in the high-resolution experiment along with theoretical modeling allowed us to relate this unusual behavior to the influence of ground-state vibrational modes, located solely on the carotenoid. After the role of vibronic coupling on $\mathrm{S}_{2}$-to- $\mathrm{Q}_{\mathrm{x}}$-transfer has been clarified in this study, future work will focus on high-time-resolution visible excitation-NIR probe experiments. These may further clarify whether or not the ultrafast $\mathrm{Q}_{\mathrm{x}}$-to- $\mathrm{Q}_{\mathrm{y}}$-transfer [44 fs in LH1 from Rhodospirillum rubrum (Maiuri et al. 2015) is vibronically mediated.

\section{Experimental}

\section{Sample preparation}

Phaeospirillum (Ph.) molischianum was cultured strictly anaerobically at $30^{\circ} \mathrm{C}$ under incandescent illumination. Cultures were harvested by centrifugation and resuspended in $50 \mathrm{mM}$ TRICIN buffer (pH 8.0). Cells were broken in a FRENCH press at 950 PSI. Membranes were resuspended in $20 \mathrm{mM}$ TRICIN-buffer ( $\mathrm{pH} 8.0$ ) to an OD of 50 at $846 \mathrm{~nm}$. The detergent $\beta$-dodecyl maltoside ( $\beta$-DDM, GLYCON, Luckenwalde, Germany) was added to $0.5 \%$; the suspension was incubated with shaking at RT for 20 min and centrifuged to remove undissolved membranes. The supernatant was loaded onto a $25-10 \%$ sucrose gradient, containing $0.03 \% \beta$-DDM and ultracentrifuged at $130.000 \mathrm{~g}$ for $16 \mathrm{~h}$ (overnight) at $4{ }^{\circ} \mathrm{C}$. Two major brownish bands were visible, the upper one being $\mathrm{LH} 2$. The $\mathrm{LH} 2$ fraction was concentrated in CENTRICON 100 devices at $20^{\circ} \mathrm{C}$ and $3.000 \mathrm{~g}$ to remove small(er) proteins. Further purification 
was carried out by another sucrose gradient and/or size exclusion chromatography on a column equilibrated with $20 \mathrm{mM}$ TRICIN-buffer (pH 8.0) and 0.03\% $\beta$-DDM.

\section{Spectroscopy}

The broadband pump-probe set-up (Polli et al. 2007) is based on an amplified Ti:sapphire laser system (Libra, Coherent), which delivers a $2 \mathrm{kHz}$ pulse train of $4-\mathrm{mJ}$, 100 -fs pulses at $800 \mathrm{~nm}$. A portion of the laser output was used to pump an optical parametric amplifier (OPA): a narrowband pulse (10 nm FWHM, $15 \mathrm{~nJ} /$ pulse, centered at $520 \mathrm{~nm}$ ) is obtained by amplification of a spectrally filtered white-light continuum generated in a $2 \mathrm{~mm}$ Sapphire plate. The pump for this process is the second harmonic of a part of the $800 \mathrm{~nm}$ beam. The white-light continuum probe pulse was generated by focusing a small fraction of the laser fundamental in a 2-mm sapphire plate. Pump and probe beams were synchronized by a delay line and focused on the sample by a $500-\mathrm{mm}$ focal length lens and a $200-\mathrm{mm}$ focal length spherical mirror, respectively. The pump beam was blocked after the sample by an iris. The total instrument response function of the system was approximately 120 fs FWHM, largely limited by the pump pulse duration.

The $\Delta \mathrm{T} / T$ differential signal was obtained by dispersing the probe pulse on an optical multichannel analyzer and subtracting pump on and pump off spectra. A 2D map of the $\Delta \mathrm{T} / T$ signal versus probe delay was obtained by stacking the signal acquired for different delays $\tau$ between pump and probe pulses.

The broadband femtosecond transient absorption data were collected using heterodyned transient grating as described previously (Lincoln et al. 2016) with the exception that the data were collected along a single phase matching direction and phased to pump probe data. The pulse spectrum, as shown in Fig. 6a, was compressed to $10 \mathrm{fs}$ duration, using a combination of a prism pair and a grating compressor in a $4 \mathrm{f}$ design.

Acknowledgements Open access funding provided by TU Wien (TUW). The authors thank Adrian Kölsch for preparing Fig. 1. ET, CNL, JH, VP, HL, and FS acknowledge Czech-Austrian Mobility (Cz: MSMT Grant No. 7AMB16AT023, Aus: OeAD for funding via the WTZ-project CZ 09/2016.). VP and FS acknowledge support of the Czech Grant Agency (GA14-25752S). GC acknowledges support by the European Research Council Advanced Grant STRATUS (ERC-2011-AdG No. 291198). GC and JH acknowledge funding by Laserlab-Europe (EU-H2020 654148). HL acknowledges support by the Grant Agency of the Czech Republic, GACR (\#P501/12/G055).

Open Access This article is distributed under the terms of the Creative Commons Attribution 4.0 International License (http:// creativecommons.org/licenses/by/4.0/), which permits unrestricted use, distribution, and reproduction in any medium, provided you give appropriate credit to the original author(s) and the source, provide a link to the Creative Commons license, and indicate if changes were made.

\section{References}

Andersson PO, Gillbro T (1995) Photophysics and dynamcis of the lowest excited singlet-state in long susbtituted polyenes with implications to the very long-chain limit. J Chem Phys 103(7):2509-2519

Balevicius V, Pour AG, Savolainen J, Lincoln CN, Lukes V, Riedle E, Valkunas L, Abramavicius D, Hauer J (2015) Vibronic energy relaxation approach highlighting deactivation pathways in carotenoids. Phys Chem Chem Phys 17(29):19491-19499. doi:10.1039/C5CP00856E

Balevičius V, Abramavicius D, Polívka T, Galestian Pour A, Hauer J (2016) A unified picture of $\mathrm{S}^{*}$ in carotenoids. J Phys Chem Lett 7(7):3347-3352. doi:10.1021/acs.jpclett.6b01455

Billsten HH, Herek JL, Garcia-Asua G, Hashoj L, Polivka T, Hunter CN, Sundstrom V (2002a) Dynamics of energy transfer from lycopene to bacteriochlorophyll in genetically-modified LH2 complexes of Rhodobacter sphaeroides. Biochemistry 41 (12):4127-4136. doi:10.1021/bi011741v

Billsten HH, Zigmantas D, Sundstrom V, Polivka T (2002b) Dynamics of vibrational relaxation in the S-1 state of carotenoids having 11 conjugated $\mathrm{C}=\mathrm{C}$ bonds. Chem Phys Lett 355(5-6):465-470

Blankenship RE (2014) Molecular mechanisms of photosynthesis, 2nd edn. Wiley, Chichester

Christensson N, Milota F, Nemeth A, Sperling J, Kauffmann HF, Pullerits T, Hauer J (2009) Two-dimensional electronic spectroscopy of beta-carotene. J Phys Chem B 113(51):16409-16419. doi:10.1021/jp906604j

Christensson N, Milota F, Nemeth A, Pugliesi I, Riedle E, Sperling J, Pullerits T, Kauffmann HF, Hauer J (2010) Electronic doublequantum coherences and their impact on ultrafast spectroscopy: the example of beta-carotene. J Phys Chem Lett 1(23):33663370. doi:10.1021/jz101409r

Christiana R, Miki T, Kakitani Y, Aoyagi S, Koyama Y, Limantara L (2009) Energies and excited-state dynamics of $1 \mathrm{~B}(\mathrm{u})(+), 1 \mathrm{~B}(\mathrm{u})$ $(-)$ and $3 \mathrm{~A}(\mathrm{~g})(-)$ states of carotenoids bound to $\mathrm{LH} 2$ antenna complexes from purple photosynthetic bacteria. Chem Phys Lett 480(4-6):289-295. doi:10.1016/j.cplett.2009.08.074

Cong H, Niedzwiedzki DM, Gibson GN, LaFountain AM, Kelsh RM, Gardiner AT, Cogdell RJ, Frank HA (2008) Ultrafast timeresolved carotenoid to-bacteriochlorophyll energy transfer in LH2 complexes from photosynthetic bacteria. J Phys Chem B 112(34):10689-10703. doi:10.1021/Jp711946w

Damjanovic A, Ritz T, Schulten K (1999) Energy transfer between carotenoids and bacteriochlorophylls in light-harvesting complex II of purple bacteria. Phys Rev E 59(3):3293-3311. doi:10.1103/ PhysRevE.59.3293

Fuller FD, Pan J, Gelzinis A, Butkus V, Senlik SS, Wilcox DE, Yocum CF, Valkunas L, Abramavicius D, Ogilvie JP (2014) Vibronic coherence in oxygenic photosynthesis. Nat Chem 6(8):706-711

Gradinaru CC, Kennis JTM, Papagiannakis E, van Stokkum IHM, Cogdell RJ, Fleming GR, Niederman RA, van Grondelle R (2001) An unusual pathway of excitation energy deactivation in carotenoids: singlet-to-triplet conversion on an ultrafast timescale in a photosynthetic antenna. Proc Natl Acad Sci USA 98(5):2364-2369

Herek JL, Wendling M, He Z, Polivka T, Garcia-Asua G, Cogdell RJ, Hunter CN, van Grondelle R, Sundstrom V, Pullerits T (2004) Ultrafast carotenoid band shifts: experiment and theory. J Phys Chem B 108(29):10398-10403 
Ihalainen JA, Linnanto J, Myllyperkio P, van Stokkum IHM, Ucker B, Scheer H, Korppi-Tommola JEI (2001) Energy transfer in LH2 of Rhodospirillum molischianum, studied by subpicosecond spectroscopy and configuration interaction exciton calculations. J Phys Chem B 105(40):9849-9856. doi:10.1021/jp010921b

Jumper CC, Arpin PC, Turner DB, McClure SD, Rafiq S, Dean JC, Cina JA, Kovac PA, Mirkovic T, Scholes GD (2016) Broad-band pump-probe spectroscopy quantifies ultrafast solvation dynamics of proteins and molecules. J Phys Chem Lett 7(22):4722-4731. doi:10.1021/acs.jpclett.6b02237

Knox RS (1999) Ultrashort processes and biology. J Photoch Photobio B 49(2-3):81-88. doi:Doi:10.1016/S1011-1344(99)00060-3

Koepke J, Hu XC, Muenke C, Schulten K, Michel H (1996) The crystal structure of the light-harvesting complex II (B800-850) from Rhodospirillum molischianum. Structure 4(5):581-597

Kosumi D, Fujiwara M, Fujii R, Cogdell RJ, Hashimoto H, Yoshizawa M (2009) The dependence of the ultrafast relaxation kinetics of the S-2 and S-1 states in beta-carotene homologs and lycopene on conjugation length studied by femtosecond timeresolved absorption and Kerr-gate fluorescence spectroscopies. J Chem Phys 130(21). doi:10.1063/1.3147008

Krueger BP, Scholes GD, Fleming GR (1998) Calculation of couplings and energy-transfer pathways between the pigments of LH2 by the ab initio transition density cube method. J Phys Chem B 102(27):5378-5386

Liebel M, Schnedermann C, Wende T, Kukura P (2015) Principles and applications of broadband impulsive vibrational spectroscopy. J Phys Chem A 119(36):9506-9517. doi:10.1021/acs. jpca.5b05948

Lincoln CN, Hayden J, Pour AG, Perlik V, Sanda F, Hauer J (2016) A quantitative study of coherent vibrational dynamics probed by heterodyned transient grating spectroscopy. Vib Spectrosc 85:167-174. doi:10.1016/j.vibspec.2016.04.018

Macpherson AN, Arellano JB, Fraser NJ, Cogdell RJ, Gillbro $T$ (2001) Efficient energy transfer from the carotenoid S-2 state in a photosynthetic light-harvesting complex. Biophys J 80(2):923-930

Maiuri M, Rehault J, Carey AM, Hacking K, Garavelli M, Luer L, Polli D, Cogdell RJ, Cerullo G (2015) Ultra-broadband 2D electronic spectroscopy of carotenoid-bacteriochlorophyll interactions in the LH1 complex of a purple bacterium. J Chem Phys 142(21). doi:10.1063/1.4919056

Mcdermott G, Prince SM, Freer AA, Hawthornthwaitelawless AM, Papiz MZ, Cogdell RJ, Isaacs NW (1995) Crystal-structure of an integral membrane light-harvesting complex from photosynthetic bacteria. Nature 374(6522):517-521. doi:10.1038/374517a0

Merlin JC (1985) Resonance raman-spectroscopy of carotenoids and carotenoid-containing systems. Pure Appl Chem 57(5):785-792. doi: $10.1351 / p a c 198557050785$

Mirkovic T, Ostroumov E, Anna JM, Van Grondelle R, Govindjee, Scholes GD (2017) Light absorption and energy transfer in the antenna complexes of photosynthetic organisms. Chem Rev 117(2):44

Niedzwiedzki DM, Kobayashi M, Blankenship RE (2011) Triplet excited state spectra and dynamics of carotenoids from the thermophilic purple photosynthetic bacterium Thermochromatium tepidum. Photosynth Res 107(2):177-186. doi:10.1007/ s11120-011-9620-x

Niedzwiedzki DM, Bina D, Picken N, Honkanen S, Blankenship RE, Holten D, Cogdell RJ (2012) Spectroscopic studies of two spectral variants of light-harvesting complex 2 (LH2) from the photosynthetic purple sulfur bacterium Allochromatium vinosum. Bba-Bioenergetics 1817(9):1576-1587. doi:DOI:10.1016/j. bbabio.2012.05.009
Niedzwiedzki DM, Hunter CN, Blankenship RE (2016) Evaluating the nature of so-called $\mathrm{S}^{*}$-state feature in transient absorption of carotenoids in light-harvesting complex 2 (LH2) from purple photosynthetic bacteria. J Phys Chem B 120(43):11123-11131. doi:10.1021/acs.jpcb.6b08639

Perlik V, Sanda F (2016) Simulations of coherent nonlinear optical response of molecular vibronic dimers. arXiv:160400327 [physicschem-ph]

Perlik V, Seibt J, Cranston LJ, Cogdell RJ, Lincoln CN, Savolainen J, Sanda F, Mancal T, Hauer J (2015) Vibronic coupling explains the ultrafast carotenoid-to-bacteriochlorophyll energy transfer in natural and artificial light harvesters. J Chem Phys 142(21). doi:10.1063/1.4919548

Polivka T, Sundstrom V (2004) Ultrafast dynamics of carotenoid excited states-from solution to natural and artificial systems. Chem Rev 104(4):2021-2071. doi:10.1021/cr020674n

Polivka T, Sundstrom V (2009) Dark excited states of carotenoids: consensus and controversy. Chem Phys Lett 477(1-3):1-11. doi:10.1016/j.cplett.2009.06.011

Polivka T, Zigmantas D, Herek JL, He Z, Pascher T, Pullerits T, Cogdell RJ, Frank HA, Sundstrom V (2002) The carotenoid S-1 state in LH2 complexes from purple bacteria Rhodobacter sphaeroides and Rhodopseudomonas acidophila: S-1 energies, dynamics, and carotenoid radical formation. J Phys Chem B 106(42):11016-11025. doi:10.1021/jp025752p

Polivka T, Niedzwiedzki D, Fuciman M, Sundstrom V, Frank HA (2007) Role of B800 in carotenoid-bacteriochlorophyll energy and electron transfer in LH2 complexes from the purple bacterium Rhodobacter sphaeroides. J Phys Chem B 111(25):74227431. doi:10.1021/jp071395c

Polli D, Cerullo G, Lanzani G, De Silvestri S, Hashimoto H, Cogdell RJ (2006) Carotenoid-bacteriochlorophyll energy transfer in LH2 complexes studied with 10 -fs time resolution. Biophys $\mathbf{J}$ 90(7):2486-2497. doi:10.1529/biophysj.105.069286

Polli D, Luer L, Cerullo G (2007) High-time-resolution pumpprobe system with broadband detection for the study of time-domain vibrational dynamics. Rev Sci Instrum 78(10). doi:10.1063/1.2800778

Rondonuwu FS, Yokoyama K, Fujii R, Koyama Y, Cogdell RJ, Watanabe Y (2004) The role of the $1(1) \mathrm{B}(\mathrm{u})(-)$ state in carotenoidto-bacteriochlorophyll singlet-energy transfer in the LH2 antenna complexes from Rhodobacter sphaeroides G1C, Rhodobacter sphaeroides 2.4.1, Rhodospirillum molischianum and Rhodopseudomonas acidophila. Chem Phys Lett 390(4-6):314-322. doi:10.1016/j.cplett.2004.03.089

Shreve AP, Trautman JK, Frank HA, Owens TG, Albrecht AC (1991) Femtosecond energy-transfer processes in the B800-850 light-harvesting complex of Rhodobacter sphaeroides-2.4.1. Biochim Biophys Acta 1058(2):280-288. doi:10.1016/ S0005-2728(05)80248-8

Theiss C, Leupold D, Moskalenko AA, Razjivin AP, Eichler HJ, Lokstein H (2008) Femtosecond spectroscopy of native and carotenoidless purple-bacterial LH2 clarifies functions of carotenoids. Biophys J 94(12):4808-4811. doi:10.1529/biophysj.107.121681

Tretiak S, Middleton C, Chernyak V, Mukamel S (2000) Bacteriochlorophyll and carotenoid excitonic couplings in the LH2 system of purple bacteria. J Phys Chem B 104(40):9540-9553

Valkunas L, Abramavicius D, Mančal Ts (2013) Molecular excitation dynamics and relaxation: quantum theory and spectroscopy. Wiley-VCH, Weinheim

van Stokkum IHM, Larsen DS, van Grondelle R (2004) Global and target analysis of time-resolved spectra. Bba-Bioenergetics. 1657(2-3):82-104 